\title{
Risk factors for complicated diverticulitis: systematic review and meta-analysis
}

\author{
H. E. Bolkenstein ${ }^{1}$ • B. J. M. van de Wall $^{2}$ • E. C. J. Consten ${ }^{1}$ • I. A. M. J. Broeders ${ }^{1}$ • \\ W. A. Draaisma ${ }^{1}$
}

Accepted: 25 July 2017 /Published online: 10 August 2017

(C) The Author(s) 2017. This article is an open access publication

\begin{abstract}
Purpose The aim of this systematic review is to identify risk factors that can predict complicated diverticulitis. Uncomplicated diverticulitis is a self-limiting and mild disease, but $10 \%$ of patients with diverticulitis develop complications requiring further treatment. It is important to estimate the risk of developing complicated diverticulitis at an early stage to set the right treatment at initial presentation.

Methods Embase, MEDLINE, and Cochrane databases were searched for studies reporting on risk factors for complicated diverticulitis. Complicated diverticulitis was defined as Hinchey $\geq \mathrm{Ib}$ or severe diverticulitis according to the Ambrosetti criteria. Meta-analyses were performed when at least four studies reported on the outcome of interest. This study was conducted according to the PRISMA guidelines.

Results A total of 12 studies were included with a total of 4619 patients. Most were of reasonable quality. Only the risk factors "age" and "sex" were eligible for meta-analysis, but none showed a significant effect on the risk for complicated diverticulitis. There was reasonable quality of evidence suggesting that high C-reactive protein; white blood cell count; clinical signs including generalized abdominal pain, constipation and vomiting; steroid usage; a primary episode; and comorbidity are risk factors for complicated diverticulitis.
\end{abstract}

H. E. Bolkenstein

he.bolkenstein@meandermc.nl

1 Department of Surgery, Meander Medisch Centrum, 3813 TZ, Amersfoort, Netherlands

2 Department of Surgery, Jeroen Bosch Ziekenhuis, Den Bosch, Netherlands
Conclusion Although high-level evidence is lacking, this study identified several risk factors associated with complicated diverticulitis. Individually, these risk factors have little value in predicting the course of diverticulitis. The authors propose a prognostic model combining these risk factors which might be the next step to aid the physician in predicting the course of diverticulitis and setting the right treatment at initial presentation.

Keywords Diverticulitis $\cdot$ Acute $\cdot$ Complicated $\cdot$ Risk factors $\cdot$ Score

\section{Introduction}

In the Netherlands, approximately 22,000 patients per year are referred to secondary care with diverticulitis [1,2]. Ten percent of these patients will develop complications such as abscess or perforation and require further treatment in the form of close observation, antibiotics, percutaneous drainage, or surgery. Uncomplicated diverticulitis is however a self-limiting and relatively mild disease [3,4]. Recent literature has indicated that the outpatient treatment of uncomplicated diverticulitis is safe and effective [5, 6]. This implies that uncomplicated diverticulitis can be safely treated in primary care. The National Guideline for general practitioners (NHG standard) considers diverticulitis a clinical diagnosis based on the following signs: the development of persistent sharp, stabbing pain in the lower left abdomen within a couple of days and pressure or rebound tenderness only in the lower left abdomen. CRP level above $20 \mathrm{mg} / \mathrm{L}$ and body temperature $>38.0{ }^{\circ} \mathrm{C}$ could support the diagnosis. Ultrasound or CT scan is deemed unnecessary in the primary care setting when the abovementioned symptoms are present. The NHG standard advises only to refer patients with a suspicion of complicated diverticulitis to secondary care [1]. 
However, a considerable amount of patients with uncomplicated diverticulitis is still referred to secondary care, resulting in unnecessary diagnostics (ultrasound, CT scan) and treatment (antibiotics, hospital admittance). To reduce the annual healthcare costs of diverticulitis and improve diverticulitis care, these unnecessary referrals should be reduced. Such a strategy would demand a proper prognostic tool to help estimate the risk of developing complicated diverticulitis, since this estimation will influence the course of action of the treating physician. If the treating physician can more accurately predict the course of the disease after setting the diagnosis, he will feel more comfortable to treat patients at home. To this day, there are no prognostic models that can predict the severity of diverticulitis. More evidence on risk factors for complicated diverticulitis is needed to establish such a model and aid the treating physician in predicting the course of diverticulitis and setting the right treatment at initial presentation. Therefore, a systematic review and meta-analysis were performed to identify risk factors for complicated diverticulitis.

\section{Material and methods}

\section{Search strategy}

This systematic review was conducted according to the Preferred Reporting Items for Systematic Reviews and Metaanalysis (PRISMA) guidelines and was executed in May 2016 [7]. The databases PubMed, Embase, and Cochrane library were searched using synonyms for domain (diverticulitis), determinant (risk factors), and outcome (complicated diverticulitis). The used search terms are listed in appendix 1. The search results were filtered for doubles, and the remaining articles were screened for title and abstract. All studies that did not report on the domain (diverticulitis) and outcome (complicated diverticulitis) were excluded. All non-English publications and studies performed before 1990 were also excluded.

The remaining articles were read for full text. Only studies comparing patients with uncomplicated to complicated diverticulitis were included in this review. Case-reports, expertopinions, reviews, and studies on right-sided diverticulitis were excluded. The references of all selected studies were hand-searched for other relevant studies. Ambiguities were resolved by consultation with the senior authors.

\section{Data extraction}

Data regarding study characteristics and all relevant risk factors were extracted. A risk factor for complicated diverticulitis encompassed all patient characteristics (medical history, age, gender, body mass index, race), clinical signs (pain, nausea, vomiting, rectal bleeding, diarrhea, constipation), physical signs (guarding, palpable mass, signs of bowel obstruction), vital signs (body temperature, heart rate, blood pressure, respiratory rate), and laboratory parameters (C-reactive protein (CRP) white blood cell (WBC) count, sodium).

Uncomplicated diverticulitis was defined as Hinchey Ia diverticulitis or "mild diverticulitis" according to the Ambrosetti classification. Complicated diverticulitis was defined as Hinchey $\geq \mathrm{Ib}$ or severe diverticulitis according to the Ambrosetti criteria (see appendix 2 and 3).

\section{Critical appraisal}

All selected articles were critically appraised by H. Bolkenstein. Cross-sectional studies were assessed on relevance and quality using the cross-sectional appraisal tool from wordpress.com [8]. Longitudinal studies were assessed using the Scottish Intercollegiate Guidelines Network (SIGN) Methodology Checklist http://www.sign.ac.uk/pdf/sign50annexc.pdf. Studies that were considered poorly were excluded to ensure the quality of the systematic review and minimize risk of confounding and bias. Ambiguities were resolved by consultation with the senior authors.

\section{Data analysis}

Review Manager (RevMan) software version 5.1 was used for the meta-analysis. Pooling of data was only performed of studies reporting mean and standard deviation and when at least four studies reported on the outcome of interest. The remaining outcomes were described qualitatively. Pooled risk ratios (RRs) comparing uncomplicated diverticulitis to complicated diverticulitis were calculated using a random effects model allowing for variation beyond chance in estimates across studies. The I 2 statistic was used to quantify the amount of heterogeneity. To obtain insight on the absolute cumulative risk of determinants, the authors used the average risk across studies.

\section{Results}

\section{Search and critical appraisal}

Search results and study selection are described in Fig. 1. A total of twelve articles were critically appraised [9-20]. Most studies were rated as reasonable to good quality based on the cross-sectional appraisal tool from wordpress.com and the SIGN Methodology Checklist [8] http://www.sign.ac.uk/ pdf/sign50annexc.pdf. Studies were mostly downgraded due to lack of control of possible confounders. None of the studies were graded high quality since the studies were of retrospective design and/or had small patient numbers and/ or poor presentation of results. The results of the critical appraisal are depicted in Table 1. 
Fig. 1 Search results

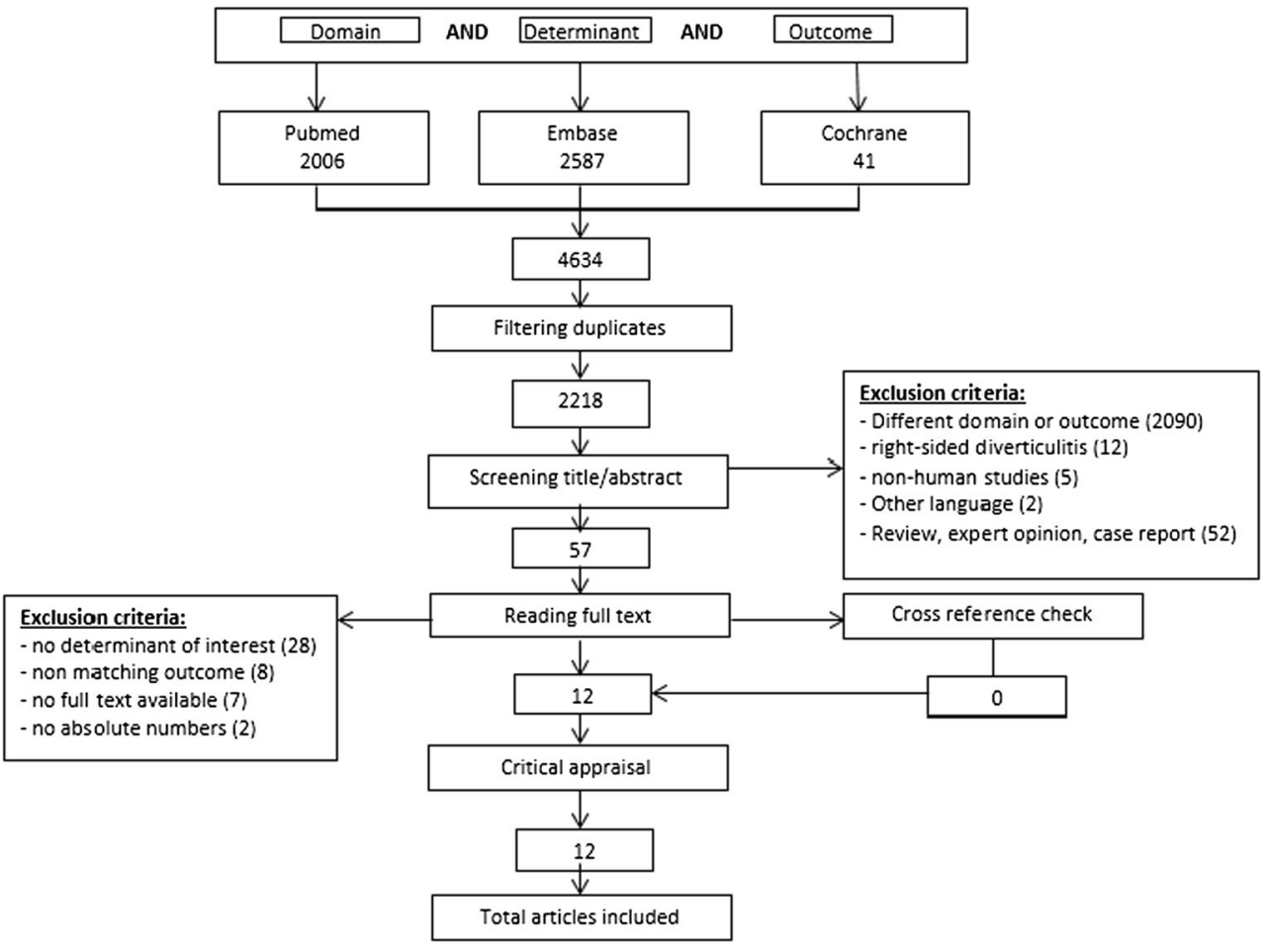

\section{Baseline characteristics}

Study characteristics of the included studies are shown in Table 1. Of the twelve included studies, four had a retrospective cross-sectional design $[14-16,19]$ and seven had a retrospective cohort design $[9-13,17,20]$. There was one prospective cross-sectional study [18]. The 12 included articles evaluated a total of 4619 patients with diverticulitis. In all studies, the diagnosis (complicated) diverticulitis was proven by computed tomography (CT) or pathological examination. A total of 3661 (79\%) patients had uncomplicated diverticulitis and 958 (21\%) had complicated diverticulitis.

\section{Main outcome—risk factors for complicated diverticulitis}

Age

Ten studies reported on age as a risk factor for complicated diverticulitis $[10-17,19,20]$. A pooled data analysis was performed on studies that reported age as a dichotomous variable (older or younger than 50 years) $[10-13,17,20]$. The pooled analysis showed no significant difference. The pooled risk ratio was 0.74 (95\% CI $0.27-2.02)$ in a random effects model $\left(I^{2}=95 \%\right)$, as depicted in Fig. 2. Makela et al. reported the influence of age on the risk for complicated diverticulitis in three groups $(<50,50-70$, and $>70$ years). They found a significant effect of old age ( $>70$ years) on the risk of complicated diverticulitis $(p=0.008)$ [15].

Pooling of studies that described age as a continuous variable (mean and standard deviation) was not possible due to the fact some studies did not report the required standard deviation of the mean age. There was no consensus among these studies on the effect of age on the severity of diverticulitis. Van de Wall et al. found that patients with complicated diverticulitis were of a significantly $(p<0.05)$ higher age $(63.9$ years $)$ as compared with patients with an uncomplicated episode (57.1 years) [19]. Nizri et al. and Longstreth et al. however did not find a significant effect. They respectively found a mean age of 63 and 57.3 years in patients with an uncomplicated episode compared to 59.3 and 56.6 years in patients with complicated diverticulitis ( $p=0.182$ and 0.71 , respectively) $[14,16]$.

\section{Gender}

Four studies reported on gender [14-16, 19]. Pooled analysis demonstrated no significant difference in risk for complicated diverticulitis. The pooled risk ratio was 0.85 (95\% CI 0.69 $1.06)$ in a random effects model $\left(I^{2}=60 \%\right)$, as depicted in Fig. 3. The absolute risk of developing complicated diverticulitis varied from 9 to $46 \%$ in women with an estimated average of $21 \%$. In men, the absolute risk of developing complicated diverticulitis varied from 16 to $59 \%$ with an estimated average of $25 \%$.

\section{History of previous attacks}

Two studies reported on history of previous attacks as a risk factor for complicated diverticulitis [16, 19]. Nizri et al. found that a primary episode of diverticulitis was at greater risk to be accompanied by complications compared to recurrent episodes 


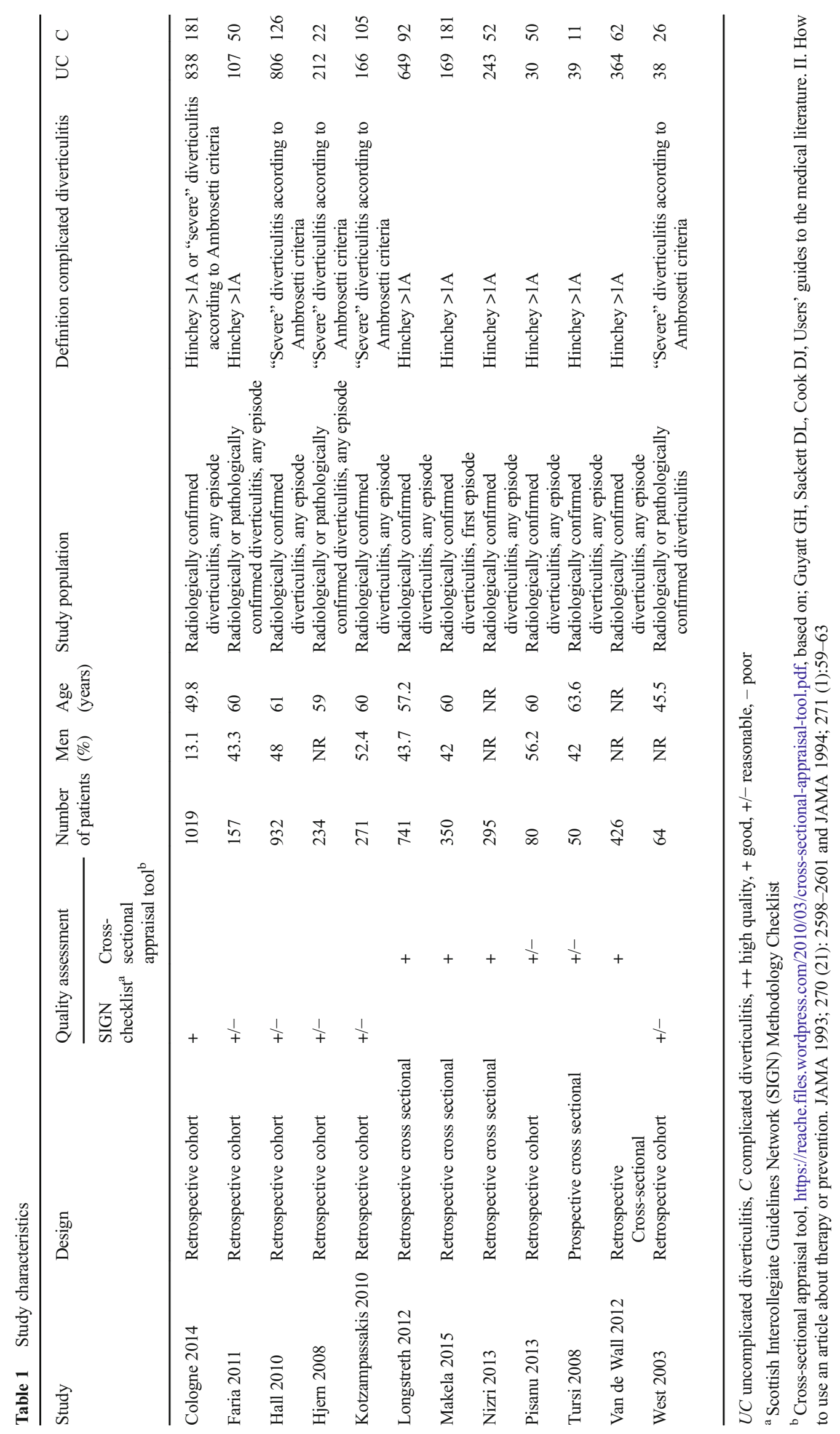


Fig. 2 Meta-analysis. Age $\leq 50$ and $>50$ years

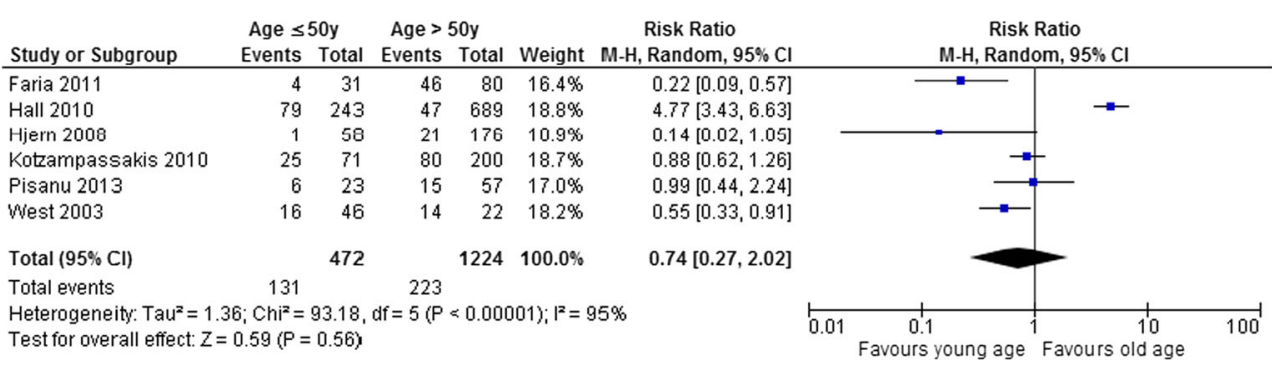

(RR 1.98, 95\% CI 1.26-3.11) [16]. Van de Wall et al. did not find a significant effect of previous attacks on the severity of diverticulitis. Twelve percent of the patients who presented with uncomplicated diverticulitis had had previous attacks, compared to $14 \%$ of the patients presenting with complicated diverticulitis [19].

\section{Clinical signs and physical examination}

Three studies reported on clinical signs (such as nausea, vomiting, bloating) as risk factors for complicated diverticulitis [14, 18, 19]. Longstreth et al. found that significantly more patients with complicated diverticulitis had signs of constipation (OR 2.32, 95\% CI 1.27-4.23). Furthermore, patients with complicated diverticulitis presented less frequently with localized pain in the lower left abdomen (OR 0.54, 95\% CI 0.29-0.99). These patients had more generalized abdominal pain [14].

Van de Wall et al. found that patients with a complicated episode presented more frequently with vomiting (26 versus $11 \%$ ) and diffuse abdominal pain (20 versus $9 \%$ ) than patients with an uncomplicated episode [19].

Tursi et al. investigated the severity of symptoms in uncomplicated and complicated diverticulitis graded on a quantitative scale. They found that patients with complicated diverticulitis had more severe constipation, abdominal pain, and, when present, more severe rectal blood loss [18].

\section{Body temperature}

Body temperature at presentation was reported in three studies. Tursi et al. found that a temperature greater than $37{ }^{\circ} \mathrm{C}$ was associated with complicated diverticulitis. Almost all patients ( 9 out of 11) with complicated diverticulitis presented with a temperature greater than $37{ }^{\circ} \mathrm{C}$ while all the patients with uncomplicated diverticulitis (39 out of 39) had a temperature below $37^{\circ} \mathrm{C}[18]$.

Longstreth et al. demonstrated that patients presenting with a temperature greater than $37.5^{\circ} \mathrm{C}$ had a higher risk of having complicated diverticulitis (OR 2.13, 95\% CI 1.27-3.57). Van de Wall reported on mean body temperature and did not find a significant effect. The mean temperature in patients with uncomplicated diverticulitis was $37.5^{\circ} \mathrm{C}(36.2-38.9)$ and $37.6^{\circ} \mathrm{C}$ (36.3-39.0) for complicated cases [14].

\section{C-reactive protein}

Four studies reported on CRP level as a risk factor for complicated diverticulitis $[15,16,18,20]$. All studies found a significant effect of CRP level on the risk of complicated diverticulitis. The overall mean CRP among patients with uncomplicated diverticulitis was $68 \mathrm{mg} / \mathrm{L}$ with a range of 25 to $96 \mathrm{mg} / \mathrm{L}$. This was $186 \mathrm{mg} / \mathrm{L}$ with a range of 134 to $224 \mathrm{mg} / \mathrm{L}$ among patients with complicated diverticulitis.

Three studies calculated the optimal threshold value of CRP level to distinguish uncomplicated from complicated diverticulitis. Makela et al. found an optimal cutoff point of $149.5 \mathrm{mg} / \mathrm{L}$ (sensitivity 65\%, specificity 85\%) [15]. The studies of Nizri et al. and van de Wall et al. found an optimal cutoff point of $90 \mathrm{mg} / \mathrm{L}$ (sensitivity 88\%, specificity 75\%) and $175 \mathrm{mg} / \mathrm{L}$ (sensitivity $61 \%$, specificity $82 \%$ ), respectively $[16,19]$.

\section{White blood cell count}

Four studies reported on this risk factor [14, 15, 18, 19]. Tursi et al. and van de Wall et al. reported on WBC as a continuous variable showing a significant effect of $\mathrm{WBC}$ on the risk of complicated diverticulitis. Average mean WBC count was $10.4 \times 10^{9} / \mathrm{L}$ (range $8.7-12.0 \times 10^{9} / \mathrm{L}$ ) in uncomplicated
Fig. 3 Meta-analysis. Sex (number of men)

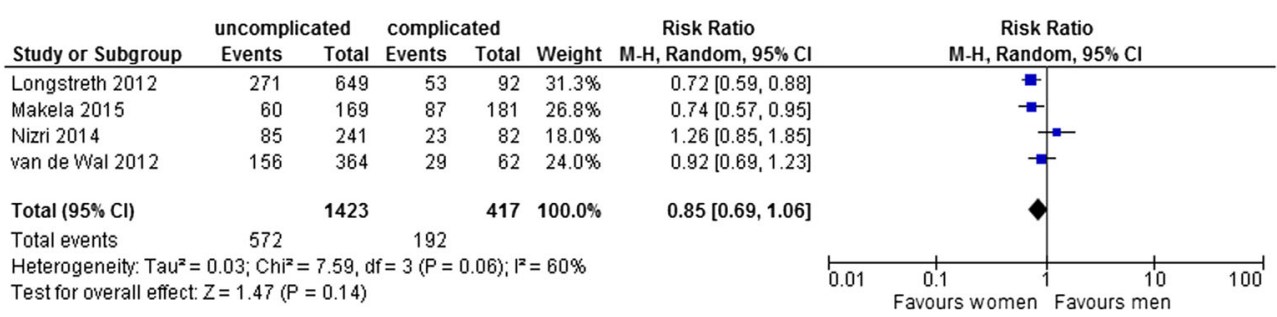


diverticulitis and $14.4 \times 10^{9} / \mathrm{L}$ (range $12.5-15.3 \times 10^{9} / \mathrm{L}$ ) in complicated diverticulitis [18, 19].

Two studies reported WBC as a dichotomous value $[14,15]$. Makela et al. reported a sensitivity of $51 \%$ and specificity of $46 \%$ for a cutoff value of $10 \times 10^{9} / \mathrm{L}(p=0.672)$ [15] Longstreth et al. found a sensitivity of $82 \%$ and specificity of $45 \%$ for a cutoff value of $11 \times 10^{9} / \mathrm{L}(p=<0.0001)$ [14].

\section{Body mass index}

Only one study reported on body mass index (BMI) as a risk factor for complicated diverticulitis. Longstreth et al. found no significant difference between patients with a BMI greater or smaller than 25 (OR 1.00 (CI 0.96-1.04) [14].

\section{Comorbidity}

One study reported on comorbidity and found that the group of patients with complicated diverticulitis consisted of patients with a higher American Society of Anesthesiologists (ASA) classification (ASA I 26\%; ASA II 65\%; ASA III 10\%) compared to the group with uncomplicated diverticulitis (ASA I 41\%; ASA II 51\%; ASA III 8\%) [19].

\section{Diabetes mellitus}

The effect of diabetes mellitus (DM) on the risk of complicated diverticulitis was reported in one retrospective cohort study. Approximately $16 \%$ of the patients without DM had complicated diverticulitis compared to $27 \%$ of the patients with DM $(p<0.003)$ [9].

\section{Steroid use and immunosuppression}

One study reported on the use of steroids. Patients with complicated diverticulitis more frequently used steroids compared to patients with uncomplicated diverticulitis ( 7.3 versus $3.3 \%$; $p=0.015)[16]$.

\section{Discussion}

\section{Summary of results}

This systematic review and meta-analysis included 12 studies with a total of 4619 patients. Few studies were found that accurately described risk factors for complicated diverticulitis. Most of the studies were of retrospective design and did not account for confounders in their analyses. The evidence in the current literature for risk factors for complicated diverticulitis is therefore not strong. Considering the high incidence of this disease and the high impact on health, quality of life, and health-care costs, this topic deserves more attention. This systematic review found that CRP, WBC count, and clinical signs (constipation, generalized abdominal pain, and vomiting) are risk factors for complicated diverticulitis. Comorbidity, number of episodes, and steroid usage were suggested as possible risk factors, but evidence for these parameters was not very strong. Only the parameters "age" and "sex" were eligible for meta-analysis. None of these parameters showed a significant difference in the risk for complicated diverticulitis.

\section{Limitations of the study}

There are some limitations of this study that should be taken into account when interpreting the results. In this systematic review, there was significant heterogeneity in the design and methods of the included studies. We accounted for this problem by using a random effects model which yields a more conservative estimate in case of heterogeneity. Additionally, we only included studies with the same definition of complicated diverticulitis, thus minimizing the heterogeneity in outcome encountered in previously published reviews.

Most risk factors were not eligible for meta-analysis since less than four studies reported them. The outcome of these risk factors could therefore only be described qualitatively and should be interpreted carefully.

\section{Interpretation of results}

We expected to find that CRP and WBC count were related to complicated diverticulitis. A higher degree of inflammation generally correlates to higher levels of inflammatory parameters. Determining the optimal threshold for use in clinical practice, however, remains a point of debate. Several studies calculated the optimal threshold for CRP level and found cutoff values ranging from 50 to $>175 \mathrm{mg} / \mathrm{L}[15,18,19]$. Optimal cutoff values for WBC count were not reported. Generally, WBC levels were higher among patients with complicated diverticulitis $\left(10.4 \times 10^{9} / \mathrm{L}\right.$ (range $\left.8.7-12.0 \times 10^{9} / \mathrm{L}\right)$ versus $14.4 \times 10^{9} / \mathrm{L}$ (range $12.5-16.3 \times 10^{9} / \mathrm{L}$ ) [18, 19].

As expected, patients with complicated diverticulitis present with more severe symptoms than patients with uncomplicated diverticulitis (diffuse versus localized abdominal pain). This could be accounted for by the presence of diffuse peritonitis $[14,18,19]$.

For the other study parameters (comorbidity, DM, BMI, history of previous attacks, steroid use), very little evidence was found. Primary episode, comorbidity, DM, and steroid use could be predictors of complicated diverticulitis [9, 16, 19]. BMI did not show a significant difference in the risk for complicated diverticulitis [14]. In general, patients with (severe) comorbidities are more prone to complications since the underlying illnesses (cardiovascular, pulmonary) could lead to impaired tissue oxygenation and perfusion, which again could lead to an increased risk of perforation. Therefore, the physician 
should be more vigilant of complicated diverticulitis in these patients. Moreover, the use of steroids abolishes the value of CRP level since steroids suppress the immune system in the inflammatory response. Physicians should take this into consideration when evaluating a patient [16].

\section{Comparison with other studies}

Recently, a systematic review of predictors of acute diverticulitis severity was published. Tan et al. concluded that comorbidity, steroid usage, first presentations, and CRP level of $>175 \mathrm{mg} / \mathrm{L}$ are predictive of a more severe disease process with higher likelihood for complications and resultant prolonged clinical course [21]. This is similar to our findings, although we found different cutoff points for CRP level (90, 149.5 , and $175 \mathrm{mg} / \mathrm{L}$ ). A major difference between Tan's review and the present review is the fact that we used one definition for complicated diverticulitis according the Hinchey classification or Ambrosetti criteria. Tan et al. employed multiple definitions such as risk of surgery, risk of medical treatment failure, and length of hospital stay. This might cause considerable heterogeneity. For this reason and the fact that the Hinchey and Ambrosetti criteria are commonly used in clinical practice, we chose to define our outcome according to these classification systems.

\section{Implication for clinical practice}

In the past decade, our understanding of diverticulitis has increased. The majority of patients have uncomplicated diverticulitis and recover without the use of antibiotics or dietary restrictions. Some studies even suggest treating these uncomplicated cases in a primary care setting [4-6]. A prognostic model would facilitate this vision and may ultimately lead to lower health-care costs for a currently costly disease with high incidence. No studies have previously attempted to develop such a model. This review aimed to identify risk factors for complicated diverticulitis, as a component of such a prognostic model. In our opinion, these risk factors individually have little discriminative value for truly estimating the risk of developing complicated diverticulitis. Combining these risk factors in a full prognostic model might be the next step to aid the treating physician in predicting the course of diverticulitis and setting the right treatment at initial presentation.

We propose a prognostic model in which the following parameters will be included: first episode of diverticulitis, vomiting, constipation, diffuse abdominal pain, rebound tenderness, défense musculaire (as a clinical sign of peritonitis), comorbidity (ASA > I), steroid usage, and CRP level (see Table 2). We chose these parameters based on the results of this systematic review and their applicability in clinical practice for primary care physicians. The weight of each parameter is based on the odds ratios, relative risks, sensitivity, specificity, negative
Table 2 Risk for complicated diverticulitis

\begin{tabular}{ll}
\hline Parameter & Score \\
\hline Anamnesis & \\
Pain & \\
$\quad$ - Localized abdominal pain & 0 \\
$\quad$ - Generalized abdominal pain & 2 \\
Constipation & 2 \\
Vomiting & 2 \\
Steroid usage & 1 \\
Patient history & \\
First episode of diverticulitis & 2 \\
ASA $\geq$ II & 1 \\
ASA $\geq$ III & 2 \\
Physical examination & \\
Rebound tenderness & 2 \\
Défense musculaire & 5 \\
Laboratory parameters & \\
CRP mg/L & \\
$-0-50$ & \\
$-50-150$ & 0 \\
$->150$ & 2 \\
\hline
\end{tabular}

Patients with total score $\geq 5$ points should be referred to secondary care

Total score: $0-4$ low risk of complicated diverticulitis, 5-8 medium risk for complicated diverticulitis, $>8$ high risk for complicated diverticulitis

predictive value (NPV), and positive predictive value (PPV) as reported by the included studies. We aimed for the highest sensitivity and NPV since the model should safely rule out complicated diverticulitis in patients suspected of acute diverticulitis. Next, we will carry out a study in a diverticulitis patient cohort to construct a nomogram to calculate the probability of complicated diverticulitis in patients with a suspicion of acute diverticulitis based on the selected parameters. By constructing a nomogram, we can evaluate and support our proposal for a clinical scoring system to make it applicable in clinical practice.

\section{Conclusion}

Although high-level evidence is lacking, this study demonstrates that CRP level, WBC count, clinical signs (generalized abdominal pain, constipation, vomiting), steroid use, number of episodes, and comorbidity are risk factors for complicated diverticulitis. Individually, these risk factors have little value for truly estimating the risk of developing complicated diverticulitis. Combining them in a prognostic model as proposed by the authors might be the next step to aid the physician in predicting the course of diverticulitis and setting the right treatment at initial presentation. 


\section{Appendix 1}

Table 3 Search terms

\begin{tabular}{|c|c|c|}
\hline Domain & Determinant & Outcome \\
\hline diverticulitis & 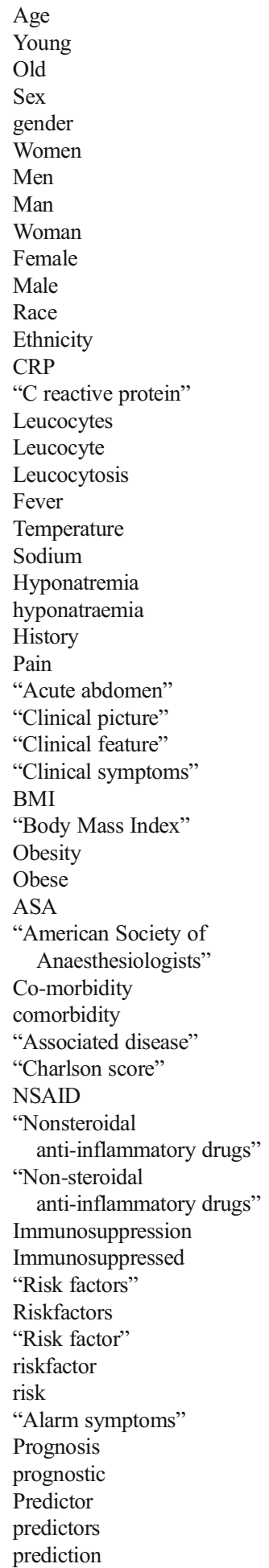 & $\begin{array}{l}\text { Complicated } \\
\text { Complication } \\
\text { Complications } \\
\text { Abscess } \\
\text { Fistula } \\
\text { Fistulae } \\
\text { Stenosis } \\
\text { "Hinchey IB" } \\
\text { "Hinchey II" } \\
\text { "Hinchey III" } \\
\text { "Hinchey IV" } \\
\text { Peritonitis } \\
\text { "Purulent peritonitis" } \\
\text { "Faecal peritonitis" } \\
\text { Perforation } \\
\text { Perforated } \\
\text { Sepsis } \\
\text { Resection } \\
\text { Surgery } \\
\text { Surgical } \\
\text { Operation } \\
\text { Operative } \\
\text { Intervention } \\
\text { Death } \\
\text { Mortality } \\
\text { "Poor outcome" }\end{array}$ \\
\hline
\end{tabular}

\section{Appendix 2}

Table 4 Hinchey classification

\begin{tabular}{|c|c|}
\hline Hinchey & Modified Hinchey \\
\hline & 0 Mild clinical diverticulitis \\
\hline \multirow[t]{2}{*}{ I Pericolic abscess or phlegmone } & $\begin{array}{l}\text { Ia Colonic wall thickening and/or } \\
\text { confined pericolic inflammation }\end{array}$ \\
\hline & $\begin{array}{l}\text { Ib Confined small pericolic abscess } \\
\quad(\leq 5 \mathrm{~cm})\end{array}$ \\
\hline $\begin{array}{l}\text { II Pelvic, distant intra-abdominal, } \\
\text { or retroperitoneal abscess }\end{array}$ & $\begin{array}{l}\text { II Pelvic, distant intra-abdominal, } \\
\text { or retroperitoneal abscess }\end{array}$ \\
\hline III Generalized purulent peritonitis & III Generalized purulent peritonitis \\
\hline \multirow[t]{3}{*}{ IV Generalized fecal peritonitis } & IV Generalized fecal peritonitis \\
\hline & Fistula \\
\hline & Obstruction \\
\hline
\end{tabular}

Hinchey EJ, Schaal PG, Richards GK. Treatment of perforated diverticular disease of the colon. Adv Surg. 1979;12: 85-109

\section{Appendix 3}

Table 5: Ambrosetti criteria

\begin{tabular}{ll}
\hline Mild diverticulitis & Severe diverticulitis \\
\hline $\begin{array}{l}\text { Localized wall thickening } \\
\text { of the bowel wall }(\geq 5 \mathrm{~mm})\end{array}$ & Bowel wall thickening $(\geq 5 \mathrm{~mm})$ \\
& and inflammation of pericolic \\
& at + one of the following: \\
Inflammation of pericolic fat & Abscess \\
& Extraluminal air \\
& Extraluminal contrast \\
\hline
\end{tabular}

Ambrosetti P, Becker C, Terrier F. Colonic diverticulitis: impact of imaging on surgical management - a prospective study of 542 patients. Eur Radiol. 2002;12:1145-1149

Open Access This article is distributed under the terms of the Creative Commons Attribution 4.0 International License (http://creativecommons.org/ licenses/by/4.0/), which permits unrestricted use, distribution, and reproduction in any medium, provided you give appropriate credit to the original author(s) and the source, provide a link to the Creative Commons license, and indicate if changes were made.

\section{References}

1. Berger MY et al (2011) NHG-standard diverticulitis. Huisarts Wet 54(9):490-498

2. Centraal Bureaau voor Statistiek. http://statline.cbs.nl/Statweb/ publication $/$ ?DM $=$ SLNL $\& \mathrm{PA}=82470$ ned $\& D 1=2 \& D 2=0 \& D 3=0$ $19 \& D 4=312 \& D 5=1 \& V W=T$. Accesed on June 2016

3. Jacobs DO (2007) Diverticulitis. N Engl J Med 357:2057-2066

4. Andeweg CS et al (2013) Guidelines of diagnostics and treatment of acute left-sided colonic diverticulitis. Dig Surg 30:278-292 
5. Biondo S et al (2014) Outpatient versus hospitalization management for uncomplicated diverticulitis. A prospective, multicenter randomized clinical trial (DIVER trial). Ann Surg 259:38-44

6. Jackson JD, Hammond T (2014) Systematic review: outpatient management of acute uncomplicated diverticulitis. J D Int J Colorectal Dis 29:775-781

7. Moher D, the PRISMA Group* et al (2009) Preferred reporting items for systematic reviews and meta-analyses: the PRISMA statement. Ann Intern Med 151:264-269

8. Cross sectional appraisal tool, https:/www.researchgate.net/file. PostFileLoader.html?id=53010affcf57d70d468b45db\&assetKey= AS\%3A272432315731969\%401441964333360, based on; Guyatt GH, Sackett DL, Cook DJ, Users' guides to the medical literature. II. How to use an article about therapy or prevention. JAMA 1993; 270 (21): 2598-2601 and JAMA 1994; 271(1):59-63. Accesed 1 June 2016

9. Cologne KG et al (2014) Effects of diabetes mellitus in patients presenting with diverticulitis. J Trauma Acute Care Surg 76(3): 704-709

10. Faria GR et al (2011) Acute diverticulitis in younger patients: any rationale for a different approach? World J Gastroenterol 17(2):207

11. Hall JF et al (2010) Colonic diverticulitis: does age predict severity of disease on CT imaging? Dis Colon Rectum 53(2):121-125

12. Hjern $\mathrm{F}$ et al (2008) Outcome of younger patients with acute diverticulitis. Br J Surg 95(6):758-764
13. Kotzampassakis $\mathrm{N}$ et al (2010) Presentation and treatment outcome of diverticulitis in younger adults: a different disease than in older patients? Dis Colon Rectum 53(3):333-338

14. Longstreth GF et al (2012) Acute diverticulitis: demographic, clinical and laboratory features associated with computed tomography findings in 741 patients. Aliment Pharmacol Ther 36:886-894. doi: 10.1111/apt.12047

15. Mäkelä JT et al (2015) The role of C-reactive protein in prediction of the severity of acute diverticulitis in an emergency unit. Scand $\mathrm{J}$ Gastroenterol 50(5):536-541

16. Nizri E et al (2014) C-reactive protein as a marker of complicated diverticulitis in patients on anti-inflammatory medications. Tech Coloproctol 18(2):145-149

17. Pisanu A et al (2013) Acute diverticulitis in the young: the same disease in a different patient. Gastroenterol Res Pract:1-6

18. Tursi A et al (2008) The clinical picture of uncomplicated versus complicated diverticulitis of the colon. Dig Dis Sci 53(9):2474 2479

19. van de Wall BJM et al (2013) The value of inflammation markers and body temperature in acute diverticulitis. Color Dis 15(5):621626

20. West SD et al (2003) Diverticulitis in the younger patient. Am J Surg 186(6):743-746

21. Tan JPL et al (2016) Predictors of acute diverticulitis severity: a systematic review. Int J Surg 26:43-52 